\title{
ENSINO DAS CÔNICAS E A PROPRIEDADE REFLEXIVA
}

Lucas Bezão Silveira Christoffer, Fernando Pereira de Souza

Universidade Federal do Mato Grosso do Sul, PET Conexões de Saberes - Matemática, Três Lagoas, MS. Email: nbezao@gmail.com

Agência de fomento: Programa de Educação Tutorial

\section{RESUMO}

O presente trabalho faz parte de uma pesquisa desenvolvida na Universidade Federal do Mato Grosso do Sul, por mim Christoffer Lucas Bezão Silveira aluno do Curso de Licenciatura em Matemática e orientado pelo Prof. Dr. Fernando Pereira de Souza. O objetivo do trabalho é explorar o ensino das Seções Cônicas e suas propriedades refletoras de maneira a desenvolver atividades diversificadas visando à construção do conhecimento, sem deixar de lado o formalismo necessário no estudo de Matemática. As atividades desenvolvidas foram elaboradas a partir de leitura e compreensão de artigos e monografias sobre o tema, os quais foram estudados através de seminários de discussão e do desenvolvimento de exercícios, e também demonstrações das construções de cada uma das cônicas. O estudo teórico pode fornecer subsídios para o desenvolvimento do conteúdo, bem como para o desenvolvimento da continuação da pesquisa.

Palavras-Chave: Cônicas, Elipse, Parábola, Hipérbole, Refletora.

\section{TEACHING BEVEL AND REFLECTIVE PROPERTY.}

\begin{abstract}
This work is part of a research developed at the Federal University of Mato Grosso do Sul, for me Christoffer Bezão Lucas Silveira student of Degree in Mathematics and supervised by Prof. Dr. Fernando Pereira de Souza. The objective is to explore the teaching of Conic Sections and their reflective properties in order to develop diverse activities aimed at the construction of knowledge, without leaving aside the necessary formalism in the study of mathematics. The activities were drawn from reading and understanding articles and monographs on the subject, which were studied by seminar discussion and development exercises, and also statements of construction of each of the conic. The theoretical study may provide a basis for the development of content as well as for the development of further research.
\end{abstract}

Keywords: Conical, Ellipse, Parable, Hyperbole, Reflector. 


\section{INTRODUÇÃO}

Pensando numa área da Matemática com muitas aplicações interessantes, o tema desenvolvido neste trabalho destina-se ao estudo das "Cônicas" que tiveram uma de suas origens no livro do Matemático e Astrônomo grego Apolônio de Perga (262 a.C - 190 a.C) com o título Cônicas, onde foram exploradas as figuras obtidas ao se cortar um cone reta por diversos planos.

Segundo Eves (2011), a obra "Cônicas", um tratado de oito volumes e aproximadamente 400 proposições, tornou famoso, já que faz um estudo exaustivo dessas curvas e supera completamente trabalhos anteriores. No Livro I de seu tratado, obtinha todas as secções cônicas da maneira hoje familiar, ou seja, a partir de um cone circular duplo, reto ou oblíquo. Os nomes elipse, parábola e hipérbole foram introduzidos por Apolônio.

O objetivo do presente trabalho é apresentar as definições das cônicas do modo de como são conhecidas e também mostrando uma propriedade das cônicas na qual se utiliza a geometria para demonstra-la.

\section{METODOLOGIA}

O trabalho foi desenvolvido através de uma série de pesquisas bibliográficas, após os estudos foram apresentados seminários para o professor orientador, na qual discutimos sobre o tema abordado e na resolução dos exercícios foram sanadas as dúvidas que surgiram durante o desenvolvimento da pesquisa.

\section{RESULTADOS}

Antes do estudo das cônicas como lugares geométricos, é interessante mostrar suas origens a partir de interseções de figuras no espaço. Desta maneira, é conveniente ilustrar que é possível gerar dois cones com o vértice comum a partir da rotação de uma reta (geratriz) ao redor de outra concorrente (eixo de rotação) como ilustrado na figura abaixo (Figura 1). Assim dependendo da inclinação de um determinado plano seccionando este cone podemos obter uma Elipse, uma hipérbole e uma parábola.

Será presentado assim as três seções cônicas elipse, hipérbole e parábola, através da sua definição e dos seus elementos.

ELIPSE

Definição: A elipse é o conjunto dos pontos do plano que satisfazem a condição: "Fixados dois pontos quaisquer do plano, $F_{1}$ e $F_{2}$, tal que, $d\left(F_{1}, F_{2}\right)=2 c, c>0$, chama-se elipse o conjunto dos pontos $P$ pertencentes ao plano cuja soma da distância entre eles, ou seja, $d\left(P, F_{1}\right)$ e $d\left(P, F_{2}\right)$ é uma constante $2 \stackrel{a}{a}, a>0$. Logo $d\left(P, F_{1}\right)+d\left(P, F_{2}\right)=2 a$.

Os elementos importantes da parábola são dados por:

- Diretriz da parábola: é a reta $r$;

- Foco da parábola: é o ponto F;

- Vértice da parábola: é o ponto $V$ sendo equidistante de $F$ e $r$;

- Parâmetro da parábola: à distância $p$ do foco à reta diretriz. 


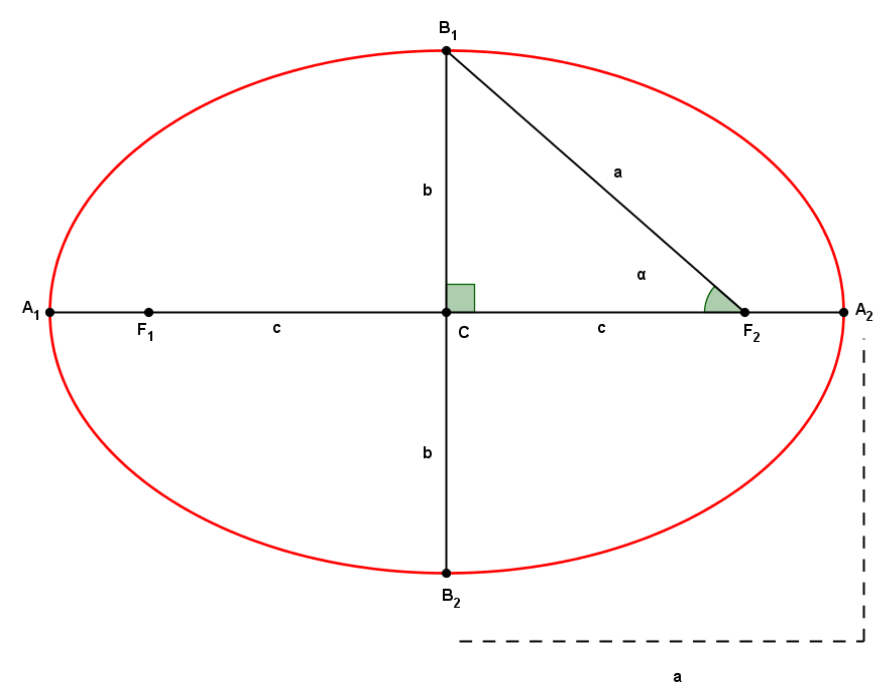

Figura 1. Elipse construída no geogebra

Assim podemos definir a elipse através de uma equação algébrica conhecida como equação reduzida da elipse que é dada por:

Observação: Os valores $a, b$ e $c$ satisfazem a relação $a^{2}=b^{2}+c^{2}$ e a equação da elipse é

$\frac{\left(x-x_{0}\right)^{2}}{a^{2}}+\frac{\left(y-y_{0}\right)^{2}}{b^{2}}=1$,

Onde, $x_{0}$ e $y_{0}$ são as coordenadas do centro $C\left(x_{0}, y_{0}\right)$ da elipse.

HIPÉRBOLE

Definição: A hipérbole é o conjunto dos pontos do plano que satisfazem a condição, fixados dois pontos $F_{1}$ e $F_{2}$ de um plano, tal que, $d\left(F_{1}, F_{2}\right)=2 c, c>0$, chama-se hipérbole o conjunto dos pontos $P$ pertencentes ao plano cujas diferenças, em módulo, das distâncias $d\left(P, F_{1}\right)$ e $d\left(P, F_{2}\right)$ é uma constante $2 a,(0<2 a<2 c)$. Logo:

$\left|d\left(P, F_{1}\right)-d\left(P, F_{2}\right)\right|=2 a$

Os elementos importantes de uma hipérbole são

dados por:

- Focos: são os pontos $F_{1}$ e $F_{2}$ da hipérbole;

- Distância focal: é a distância $2 c, c>0$ entre os

focos da hipérbole e $c$ é a semidistância focal;

- Eixo real: é o segmento $A_{1} A_{2}$ medindo $2 a$;

- Eixo imaginário: é o segmento $B_{1} B_{2}$ medindo $2 b$;

- Centro: é o ponto O médio do eixo real;

- Distância focal: é a distância $2 c$ entre os focos da

hipérbole.

- Semieixos: são os segmentos $A_{1} C, A_{2} C, B_{1} C, B_{2} C$.

- Excentricidade: é dado por $c / a$. 


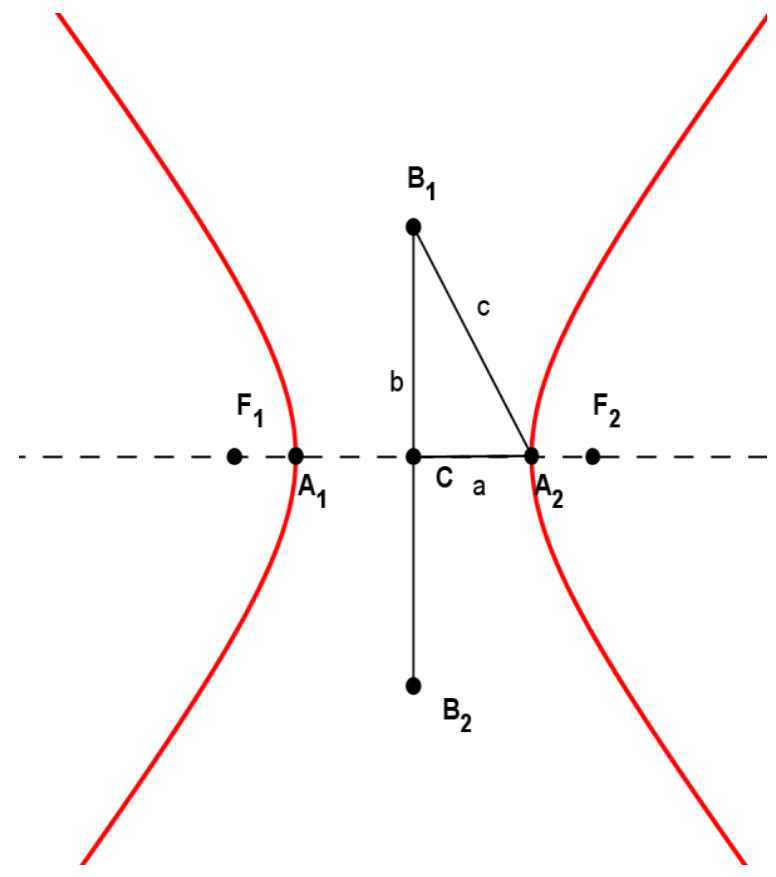

Figura 2. Hipérbole feita pelo autor no geogebra

Assim podemos definir a hipérbole através de uma equação algébrica conhecida como equação reduzida da hipérbole que é dada por:

Observação: Os valores de $a, b$ e $c$ satisfazem a relação $c^{2}=a^{2}+b^{2}$ e a equação da hipérbole é

$$
\frac{\left(x-x_{0}\right)^{2}}{a^{2}}-\frac{\left(y-y_{0}\right)^{2}}{b^{2}}=1
$$

Onde, $x_{0}$ e $y_{0}$ são as coordenadas do centro $C\left(x_{0}, y_{0}\right)$ da hipérbole.

\section{PARÁBOLA}

Definição: Dados uma reta $d$ e um ponto $F$ de tal modo que $F$ não pertencente a $d$. Define-se parábola como sendo o lugar geométrico dos pontos $P$ do plano tais que $d(P, F)=d(P$, $d)$, onde $F$ é denominado foco da parábola e a reta $r$, é chamada de diretriz.

Os elementos importantes de uma Parábola

são dados por:

- Foco: o ponto $F$ da parábola;

- Diretriz: é a reta $d$ da parábola;

- Vértice: o ponto $V$, sendo equidistante de

$F$ e de $r$;

- Eixo de simetria: é o segmento VF e o parâmetro da parábola é dado por

$$
d(F, V)=d(V, d)=P / 2 \text {. }
$$




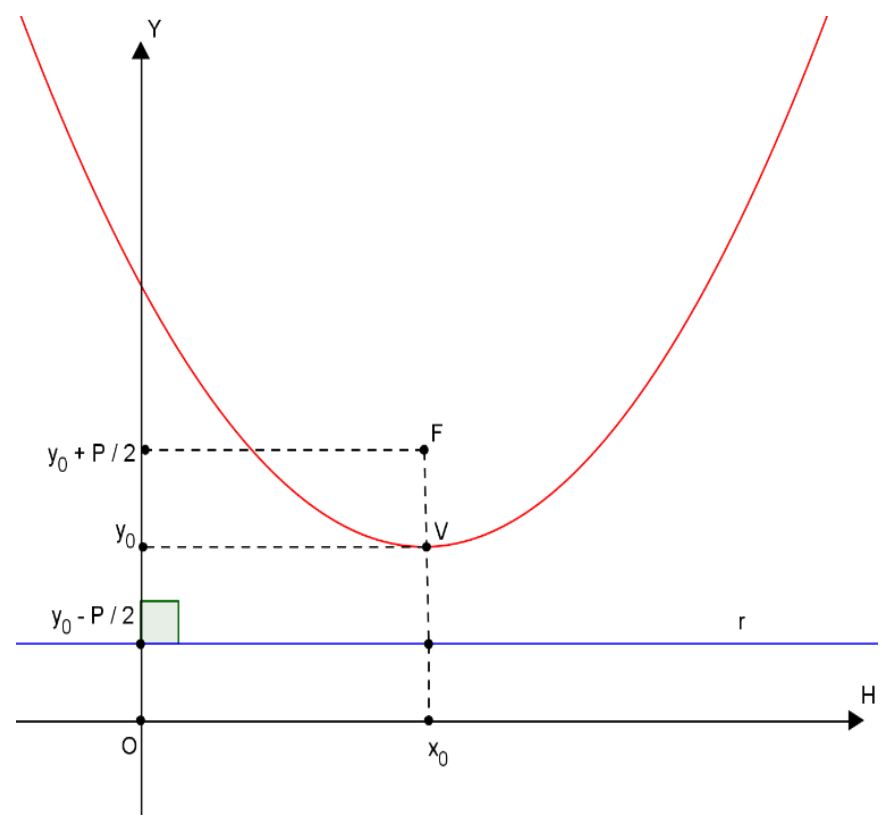

Figura 3. Parábola feita pelo autor no geogebra

Assim podemos definir a parábola através de uma equação algébrica conhecida como equação reduzida da parábola que é dada por:

$\left(y-y_{0}\right)^{2}=2 p\left(x-x_{0}\right)^{2}$,

Onde, $x_{0}$ e $y_{0}$ são as coordenadas do vértice $V\left(x_{0}, y_{0}\right)$ da parábola.

\section{PROPRIEDADES REFLETORAS DAS CÔNICAS}

Uma aplicação das cônicas é a propriedade reflexiva de cada cônicas, para se obter um melhor desenvolvimento e compreensão conceitos básicos de geometria e de física tais como os raios luminosos e as ondas sonoras propagam-se num determinado meio, em linha reta e de forma radial a partir de uma certa fonte. Se estes raios ou ondas estiverem muito afastados de seu destino estes chegam formando um feixe praticamente paralelo, como é o caso dos raios solares, por

exemplo.

Deste modo, ao incidirem num ponto de uma superfície suave, estes serão refletidos na mesma direção que refletiriam num plano que é tangente a superfície neste ponto, isto é, obedecendo a Lei da Reflexão da física onde diz que "o ângulo de incidência é igual ao ângulo de reflexão".

\section{DISCUSSÃO}

Pode ser demonstrado que cada propriedade reflexiva das cônicas, o que faremos a seguir para os casos da elipse, hipérbole e parábola.

\section{ELIPSE}

Seja uma elipse $E$ de focos $F_{1}$ e $F_{2}$, e seja $T$ um ponto de $E$. Então a reta $r$ tangente a $E$ em $T$, forma ângulo iguais $a 1$ e $a 2$ com os raios focais $F_{1} T$ e $F_{2} T$ (Propriedade bissetora da elipse). A importante consequência deste teorema, 'e que os raios luminosos ou ondas sonoras emitidas por um dos focos, ao incidirem na superfície lisa da elipse, são refletidos diretamente ao outro foco. Demonstração: Como em uma circunferência pode existir uma reta tangente, teremos uma reta $r$ tangente à elipse $E$ se está a toca em apenas um, e somente um ponto, ou seja, o ponto de tangência $T$. Estabelecendo a notação de que a distância entre dois pontos $C$ e $D$ é igual a $d(C, D)$ e a definição da elipse $E$ como o lugar geométrico dos pontos $P$ do plano que satisfazem a propriedade métrica $d\left(P, F_{1}\right)+d\left(P, F_{2}\right)=2 a=k$ (constante), pode-se concluir que: um ponto $A$ não pertence à elipse $E$ se, e somente se, $d\left(A, F_{1}\right)+d\left(A, F_{2}\right) \neq k$. Decorrente disso, uma reta $r$ é tangente à elipse $E$ em um ponto $T$ se, e somente se, tocar $E$ unicamente em $T$ (devido à tangência), e para um outro ponto $A$ qualquer de $r$, deve-se ter $T \neq A$. Isto quer dizer que: $d\left(A, F_{1}\right)+d\left(A, F_{2}\right) \neq d\left(T, F_{1}\right)$ 
$+d\left(T, F_{2}\right)$. Seja $E$ uma elipse de focos $F_{1}$ e $F_{2}$, agora toma-se um ponto $T$ na elipse e também uma reta $r$ que deve ser bissetriz de um ângulos formados pelas retas $F_{1} T$ e $F_{2} T$. Isto de tal maneira que o ângulo entre $F_{1} T$ e $r$ seja congruente ao ângulo entre $F_{2} T$ e $r$. Se mostrado que $r$ é tangente a $E$ em $T$, consequentemente, prova-se a propriedade bissetora referente ao teorema, devido à unicidade da tangente à elipse por um de seus pontos. Assim, segue que: Seja $T$ um ponto de $E$, então: $d\left(T, F_{1}\right)+d\left(T, F_{2}\right)=k$, onde $k$ é uma constante. Tomemos sobre $r$ um ponto $A$ distinto de $T$ e consideremos o $F_{1}^{\prime}$, simétrico de $F_{1}$ em relação a $r$. Sabe-se que um ponto é simétrico a outro ponto em relação a uma reta, quando são equidistantes da reta dada e estão situados sobre a mesma perpendicularidade à reta. $A$ reta $r$ é então mediatriz de $F_{1} F_{1}^{\prime}$, logo: $d\left(T, \quad F_{1}\right)=d\left(T, \quad F_{1}^{\prime}\right)$ e também, $d\left(A, \quad F_{1}\right)=d\left(A, \quad F_{1}^{\prime}\right)$. Assim pela construção a reta $r$ faz ângulos iguais com os segmentos $T F_{1}$ e $T F_{2}\left(a_{1}\right.$ e $a_{2}$ respectivamente) pois $r$ é a bissetriz. Devido à simetria, os ângulos $a_{2}$ e $a_{3}$ são consequentemente iguais também, daí os segmentos $F_{2} T$ e $T F^{\prime}{ }_{1}$ fazem ângulos congruentes com $r$ segue que: $a_{2}+\alpha+$ $a_{1}=180$ logo, $a_{3}+a_{2}+\alpha=180$ portanto os pontos $F_{1}^{\prime}, T$ e $F_{2}$ são colineares. Pela desigualdade triangular, segue-se que: $k=d\left(T, F_{1}\right)+d\left(T, F_{2}\right)=d\left(T, F_{1}^{\prime}\right)+d\left(T, F_{2}\right)=d\left(F_{1}^{\prime}, F_{2}\right)<d\left(A, F_{1}^{\prime}\right)+d\left(A, F_{2}\right)$ $=d\left(A, F_{1}\right)+d\left(A, F_{2}\right)$. Portanto, como $d\left(A, F_{1}\right)+d\left(A, F_{2}\right)>k$, conclui-se que $T$ é o único ponto de $r$ que pertence à elipse $E$, mostrando que a reta é tangente a $E$ em $T$.

\section{HIPÉRBOLE}

"O raio de luz que incide numa superfície espelhada hiperbólica direcionada a um dos focos da mesma é refletido passando pelo outro foco".

Demonstração: Seja $T$ um ponto pertencente à hipérbole de focos $F_{1}$ e $F_{2}$, como objetivo deve-se provar que a bissetriz do ângulo $F_{1} T F_{2}$ (reta $r$ ) é também tangente à hipérbole em $T$. Para isto, supõe-se que a bissetriz e a tangente sejam a mesma reta, logo: Seja $B$ um ponto pertencente a $r$ e $G$ um ponto simétrico a $F_{2}$ em relação à reta $r$, onde $G F_{2} \perp B T \perp N T$ ( $N$ é um ponto pertencente à reta normal $n$ ) donde $N T / / G F_{2}$ e observando os triângulos GTM e $F_{2} T M$ ( $M$ o ponto de intersecção do segmento de reta $G F_{2}$ e a reta $r$ ), os triângulos são congruentes por ALA (ângulo, lado, ângulo), assim temos que o triângulo $G T F_{2}$ é isósceles, como consequência disto, os ângulos do triângulo em $T G F_{2}$ e $T F_{2} G$ são iguais. Mas o ângulo $A F_{2} G$ é igual ao ângulo de incidência $A T N$, pois são correspondentes e, o ângulo $T G F_{2}$ é igual ao ângulo $N T F_{1}$, alternos internos. Assim $A T N=N T F_{1}$, ou ainda, por consequência $A T B=F_{1} T M$. Resultado que prova a Lei da Física que o ângulo de incidência é igual ao de reflexão. Agora basta provar que $B T$ é ao mesmo tempo bissetriz e tangente à hipérbole no ponto $T$. Note-se que: $B F_{1}<B G+G F_{1}$ (Desigualdade triangular), logo: $B F_{1}$ $-B F_{2}<B G+G F_{1}-B F_{2}$, mas $B G=B F_{2}$ ( $r$ também é a mediatriz assim o triângulo $B G F_{2}$ é isósceles), portanto: $B F_{1}-B F_{2}<G F_{1}=T F_{1}-T G=T F_{1}-T F_{2}=k$ (constante pela definição de hipérbole). Como a definição da hipérbole diz que o conjunto dos pontos $P$ pertencentes ao plano cuja diferença em módulo das distâncias $d\left(P, F_{1}\right)$ e $d\left(P, F_{2}\right)$ é uma constante $2 a=k$, portanto o ponto $B$ é externo ao ramo da hipérbole por $T$, ou seja, a bissetriz $B T$ toca a hipérbole apenas em $T$, logo tangente.

\section{PARÁBOLA}

"O raio de luz que incide numa superfície espelhada parabólica paralelamente ao seu eixo, é refletido em direção ao foco."

Demonstração: Consideremos agora um ponto $T$ qualquer da parábola de foco $F$, diretriz $d$ e $D$ projeção ortogonal de $T$ sobre $d$, tracemos a reta $r$, bissetriz do ângulo FTD. O Objetivo é mostrar que $r$ é tangente à parábola no ponto $T$. No triângulo $F T D$, como $F D=T D$ (pela definição de parábola), a reta $r$ comum aos triângulos $F M T$ e $D M T$ ( $M$ ponto de intersecção do seguimento $F D$ e a reta $r$ ), logo temos que os triângulos são congruentes por LAL (lado, ângulo, lado) e assim a bissetriz do ângulo $F T D$, contém a mediana, e a altura $(F M T+D M T=180$ e FMT $=D M T)$, ou seja, $r$ é a mediatriz do seguimento $F D$. 
Agora seja um ponto $Q$ qualquer da reta $r$, distinto de $T$, sendo $D^{\prime}$ a projeção ortogonal de $Q$ sobre d, daí segue que $Q F=Q D>Q D^{\prime}$ pois $Q D$ é a hipotenusa e $Q D^{\prime}$ é um cateto no triangulo $D^{\prime} Q D$, desta forma, o ponto $Q$ é exterior à parábola, ou seja, $T$ é o único ponto de $r$ que pertence à parábola. Assim agora é preciso mostrar que o ângulo de incidência é igual ao de reflexão, assim é possível observar que a semirreta $T C$, prolongamento do segmento $D T$, representa a direção dos raios incidentes. Como a tangente à parábola em $T$ é a bissetriz do ângulo, têm-se quem $a_{1}$ e $a_{2}$ são ângulos iguais com esta tangente, e como $a_{1}$ e $a_{3}$ são ângulos opostos pelo vértice, então podemos concluir que $a_{2}=a_{3}$. Desta maneira, conclui-se que todo sinal recebido paralelo ao eixo da parábola, toma a direção do foco após reflexão, como nas antenas e, todo sinal originado do foco ao refletir na superfície da parábola toma o sentido paralelo ao eixo desta.

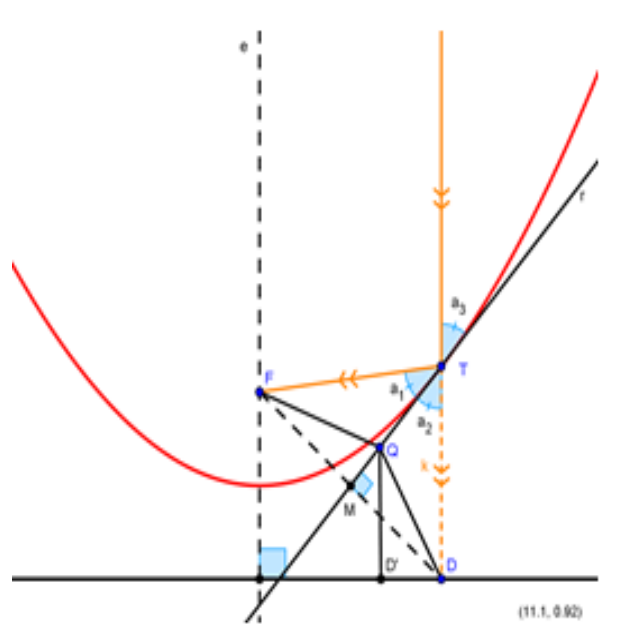

PARÁBOLA

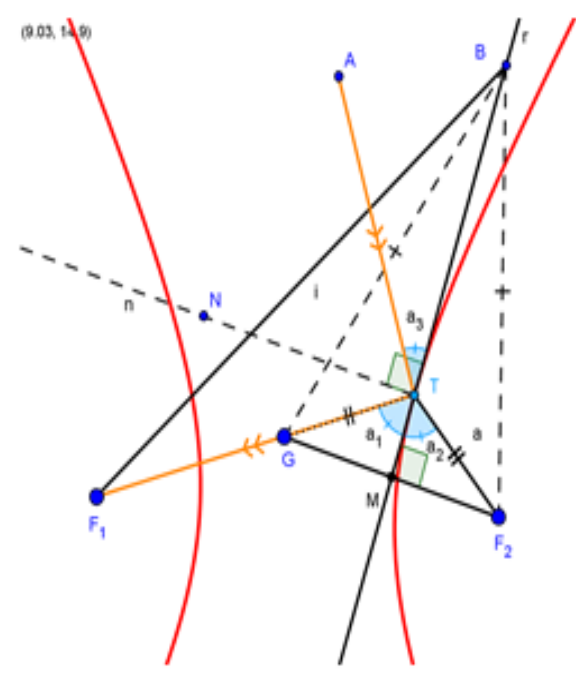

HIPÉRBOLE

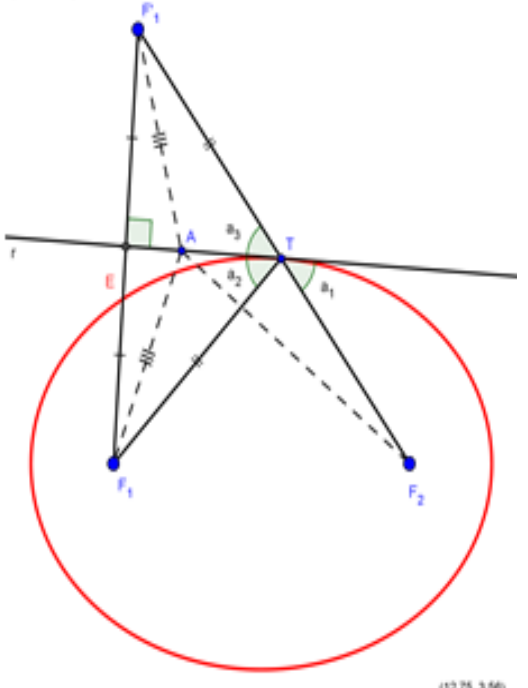

ELIPSE

\section{CONCLUSÃO}

O estudo das cônicas é importante não só para a geometria, mas também por estar conectado com outros ramos da matemática bem como a álgebra na manipulação das sentenças matemáticas. Este trabalho proporcionou um estudo abrangente sobre cônicas e suas propriedades tais como a Reflexiva tratada no presente trabalho e que não é estudado no curso de licenciatura em Matemática da UFMS campus de Três Lagoas. Podemos concluir que os objetivos foram alcançados já que o trabalho proposto foi desenvolvido e mantem a continuidade desta pesquisa científica na área.

\section{REFERENCIAS}

EVES, H. Introdução à História da Matemática. Tradução Hygino H. Domingues. 5a ed. - Campinas, SP: Editora da Unicamp, 2011.

BOULOS, P. e CAMARGO, I. Geometria Analítica - Um tratamento vetorial. 3a edição, São Paulo: Makron Books, 2007

JÚLIO CESAR CALVOSO. Estudo das cônicas com aplicações e o software geogebra como ferramenta de apoio didático. Dissertação do Programa de Mestrado Profissional em Matemática em Rede Nacional PROFMAT . Três Lagoas - MS, 2014 